\title{
Sur l'unicité de la forme des factorisations de longueur maximale d'une extension purement inséparable finie
}

El Hassane Fliouet

Résumé. Let $K / k$ be a finite purely inseparable extension of field $k$ of characteristic $p>0$. A factorization of length $m$ of $K / k$ is the data of $m$ intermediate fields $K_{1}, K_{2}, \cdots, K_{m}$ of $K / k$ such as $K \simeq K_{1} \otimes_{k} K_{2} \otimes_{k} \cdots \otimes_{k} K_{m}$. In the present paper, we are especially interested in the uniqueness of the form of factorizations of maximum length.

AMS Subject Classification (2000). 12F15

Keywords. Purement inséparable, factorisation, e-factorisation, modulaire

\section{Introduction.}

Soit $K / k$ une extension finie de caractéristique $p>0$. Une partie $G$ de $K$ est dite $r$-générateur de $K / k$, si $K=k(G)$. Si de plus, $|G|$ est minimum, $G$ est dite $r$-base de $K / k$. Soient $B_{1}$ une $r$-base de $K / k$ et $B_{2}$ une $p$-base de $k$ (c'est-à-dire $B_{2}$ est un $r$-générateur minimal de $\left.k / k^{p}\right)$. On note $\operatorname{di}(K / k)=\operatorname{card}\left(B_{1}\right)$ et $\operatorname{di}(k)=\operatorname{card}\left(B_{2}\right), \operatorname{di}(K / k)$ s'appelle le degré d'irrationalité de $K / k$ et $d i(k)$ le degré d'imperfection de $k$. Une partie $A$ de $K$ est dite $r$-libre sur $k$, si $A$ est une $r$-base de $k(A) / k$. Dans tout le reste de cette partie, on suppose que $K / k$ est purement inséparable finie. Soit $x \in K$, on pose : 
$o(x / k)=\inf \left\{m \in \mathbb{N} \mid x^{p^{m}} \in k\right\}$ et $o_{1}(K / k)=\inf \left\{m \in \mathbb{N} \mid K^{p^{m}} \subset k\right\}$. Une $r$-base $B=\left(a_{1}, a_{2}, \ldots, a_{n}\right)$ de $K / k$ est dite canoniquement ordonnée, si pour $j=1,2, \ldots, n$, on a $o\left(a_{j} / k\left(a_{1}, a_{2}, \ldots, a_{j-1}\right)\right)=o_{1}\left(K / k\left(a_{1}, a_{2}, \ldots, a_{j-1}\right)\right)$. L'entier $o\left(a_{j} / k\left(a_{1}, \ldots, a_{j-1}\right)\right)$ ainsi obtenu est indépendant du choix de la $r$-base $B$ de $K / k$ (cf. [4], p. 90, satz 14). On l'appelle le $j$-ème exposant de $K / k$, et on le note $o_{j}(K / k)$. Par convention, si $j>n$, on pose $o_{j}(K / k)=0$. On rappelle que $K / k$ est dite modulaire, s'il existe une $r$-base $\left\{a_{1}, \cdots, a_{n}\right\}$ de $K / k$ telle que $K \simeq k\left(a_{1}\right) \otimes_{k} \cdots \otimes_{k} k\left(a_{n}\right)$. Faute d'être modulaire, $K$ admet toujours une factorisation en produit tensoriel sur $k$ d'extensions irréductibles non toutes simples. Dans [1] nous avons introduit une forme particulière de factorisation définie comme suit : soient $K_{1} / k$ et $K_{2} / k$ deux sous-extensions de $K / k$ avec $d i\left(K_{1} / k\right)=i_{1}$ et $d i\left(K_{2} / k\right)=i_{2}$. Si $K \simeq K_{1} \otimes_{k} K_{2}$, et si de plus $\left(o_{1}\left(K_{1} / k\right), \cdots, o_{i_{1}}\left(K_{1} / k\right), o_{1}\left(K_{2} / k\right), \cdots, o_{i_{2}}\left(K_{2} / k\right)\right)$ est la liste des exposants de $K / k$, on dit que $K_{1} \otimes_{k} K_{2}$ est une $e$-factorisation de $K / k$. Il est facile de voir que c'est une lois associative, ce qui permet de donner un sens à une $e$-factorisation d'un nombre quelconque de facteurs sans spécifier les parenthèses. Par récurrence sur $[K: k]$, on vérifie aussitôt que $K / k$ admet une $e$-factorisation de longueur maximale. De plus, dans [1] nous avons montré que si $K_{1} \otimes_{k} \cdots \otimes_{k} K_{m}$ et $L_{1} \otimes_{k} \cdots \otimes_{k} L_{m}$ sont deux $e$-factorisations de $K / k$ avec $m$ maximal, alors pour tout $j \in\{1, \cdots, m\}$, on a $\operatorname{di}\left(K_{j} / k\right)=d i\left(L_{j} / k\right)$, et donc pour tout $j \in\{1, \cdots, m\}$, pour tout $h \in \mathbb{N}, o_{h}\left(K_{j} / k\right)=o_{h}\left(L_{j} / k\right)$. Par conséquent, dans la classe des e-factorisations de longueur maximale les facteurs conservent leurs tailles et leurs formes, donc on peut espérer étendre ce résultat à d'autres types de factorisations. Le présent papier s'inscrit dans cette direction, on s'intéresse surtout à l'unicité de la forme des factorisations de même longueur maximale. Dans ce contexte, on démontre que si $\operatorname{di}(K / k) \leq 4$ et si $K / k$ admet une $e$-factorisation de longueur maximale $m$, alors toute autre factorisation de $K / k$ de longueur m est nécessairement une $e$-factorisation. En particulier, si $2<d i(K / k) \leq 4$ et si $K / k$ admet deux types différents de factorisations de longueur $d i(K / k)-1$ dont l'une est une $e$-factorisation, alors $K / k$ est modulaire. Cependant, si $d i(K / k) \geq 5$, il existe une classe importante d'extensions qui ne conservent ni la taille ni la forme des facteurs d'une factorisation de longueur maximale. A cette occasion, on présente un exemple d'extension de degré d'irrationalité 5 admettant deux différents types de factorisations de même longueur maximale dont l'une est une $e$-factorisation.

Enfin, nous attirons l'attention que les propriétés du degré d'irrationalité, des exposants, et des extensions modulaires sont de grande importance pour ce travail. Pour cela, nous avons cru bon de rappeler ce qui est nécessaire dans la section suivante. 


\section{Terminologies et notations.}

\subsection{Degré d'irrationalité d'une extension.}

Désormais, et sauf mention expresse du contraire, $K / k$ désigne une extension algébrique finie (souvent purement inséprable) de caractéristique $p \neq 0$. Le résultat ci-dessous intervient fréquemment dans ce travail. Il permet de ramener l'étude des propriétés de " $r$-indépendance " sur $k$ aux propriétés de $p$-indépendance sur $k\left(K^{p}\right)$ lesquelles sont plus riche (théorème de la base incomplète $\cdots), K / k\left(K^{p}\right)$ étant de hauteur 1.

$\left(\mathbf{P}_{\mathbf{1}}\right)$ Soit $K / k$ une extension purement inséparable finie. Une partie $G$ de $K$ est une $r$-base de $K / k$ si, et seulement, si $G$ est une $r$-base de $K / k\left(K^{p}\right)$ (cf. [1], p. 370, proposition 1).

Notamment, nous utilisons souvent la propriété suivante :

Si $K / k$ est de hauteur $\leq 1\left(K^{p} \subseteq k\right)$, alors un système $\left(a_{1}, \cdots, a_{n}\right)$ de $K$ est $r$-libre sur $k$ si, et seulement, si $a_{i} \notin k\left(a_{1}, \cdots, a_{i-1}, a_{i+1}, \cdots, a_{n}\right)$ pour $i=1 \cdots n$.

Voici les autres résultats du degré d'irrationalité dont on a besoin.

$\left(\mathbf{P}_{\mathbf{2}}\right)$ Pour toute sous-extension $L / L^{\prime}$ de $K / k$, on a $\operatorname{di}\left(L / L^{\prime}\right) \leq \operatorname{di}(K / k)$ (cf. [1], p. 371, théorème 1).

$\left(\mathbf{P}_{\mathbf{3}}\right)$ Pour toute extension finie $K / k$, on a di $(K / k) \leq \operatorname{di}(k)$ (cf. [1], $p$. 372, théorème 2).

$\left(\mathbf{P}_{4}\right)$ Soient $K_{1} / k$ et $K_{2} / k$ deux sous-extensions de $K / k, k$-linéairement disjointes. Soient $\left\{\alpha_{1}, \cdots, \alpha_{n_{1}}\right\}$ et $\left\{\beta_{1}, \cdots, \beta_{n_{2}}\right\}$ deux $r$-bases respectivement de $K_{1} / k$ et $K_{2} / k$. On $a$ :

(i) $\left\{\alpha_{1}, \cdots, \alpha_{n_{1}}\right\}$ est une $r$-base de $K_{1}\left(K_{2}\right) / K_{2}$. En particulier, di $\left(K_{1}\left(K_{2}\right)\right.$ $\left./ K_{2}\right)=\operatorname{di}\left(K_{1} / k\right)$.

(ii) Supposons que $K_{1} / k$ et $K_{2} / k$ sont non séparables, alors $\left\{\alpha_{1}, \cdots, \alpha_{n_{1}}\right.$, $\left.\beta_{1}, \cdots, \beta_{n_{2}}\right\}$ est une $r$-base de $K_{1}\left(K_{2}\right) / k$. En outre, di $\left(K_{1}\left(K_{2}\right) / k\right)=$ $\operatorname{di}\left(K_{1} / k\right)+\operatorname{di}\left(K_{2} / k\right)$ (cf. [1], p. 371, proposition 3).

\subsection{Exposants d'une extension.}

De même, voici les principaux résultats dont on a besoin et qui font intervenir les exposants.

$\left(\mathbf{P}_{\mathbf{5}}\right)$ Soient $K$ et $L$ deux corps intermédiaires d'une extension $\Omega / k$, avec $K / k$ purement inséparable finie. Alors, pour tout entier $j$, on a $o_{j}(K(L)$ $/ k(L)) \leq o_{j}(K / k)$ (cf. [1], p. 373, proposition 5).

$\left(\mathbf{P}_{6}\right)$ Soit $K / k$ une extension purement inséparable finie. Pour toute sousextension $L / L^{\prime}$ de $K / k$, et pour tout $j \in \mathbb{N}$, on a $o_{j}\left(L / L^{\prime}\right) \leq o_{j}(K / k)$ (cf. [1], p. 374, proposition 6). 
$\left(\mathbf{P}_{\mathbf{7}}\right)$ Soient $m_{j}$ le $j$-ème exposant d'une extension purement inséparable finie $K / k$, et $\left(\alpha_{1}, \cdots, \alpha_{n}\right)$ une $r$-base canoniquement ordonnée de $K / k$. On $a$ :

(i) $k\left(K^{p^{m_{j}}}\right)=k\left(\alpha_{1}^{p^{m_{j}}}, \cdots, \alpha_{j-1}^{p^{m_{j}}}\right)$.

(ii) Soit $\Lambda_{j}=\left\{\left(i_{1}, \cdots, i_{j-1}\right)\right.$ tel que $0 \leq i_{1}<p^{m_{1}-m_{j}}, \cdots, 0 \leq i_{j-1}<$ $\left.p^{m_{j-1}-m_{j}}\right\}$. Alors, $\left\{\left(\alpha_{1}, \cdots, \alpha_{j-1}\right)^{p^{m_{j} \xi}}\right.$ tel que $\left.\xi \in \Lambda_{j}\right\}$ est une base de $k\left(K^{p^{m_{j}}}\right)$ sur $k$.

(iii) Soient $n \in \mathbb{N}$ et $j$ le plus grand entier tel que $m_{j}>n$. Alors, $\left(\alpha_{1}^{p^{n}}, \cdots, \alpha_{j}^{p^{n}}\right)$ est une $r$-base canoniquement ordonnée de $k\left(K^{p^{n}}\right) / k$, et sa liste des exposants est $\left(m_{1}-n, \cdots, m_{j}-n\right)$ (cf. [2], p. 140, proposition 5.3).

$\left(\mathbf{P}_{\mathbf{8}}\right)$ Si $K_{1} / k$ et $K_{2} / k$ sont deux sous-extensions purement inséparables de $K / k$, alors $K_{1} / k$ et $K_{2} / k$ sont $k$-linéairement disjointes si, et seulement, si pour tout $j \in \mathbb{N}, o_{j}\left(K_{1}\left(K_{2}\right) / K_{2}\right)=o_{j}\left(K_{1} / k\right)$. En particulier, si $K \simeq K_{1} \otimes_{k} K_{2}$, et si $\left(\alpha_{1}, \cdots, \alpha_{n_{1}}\right)$ est une $r$-base canoniquement ordonnée de $K_{1} / k$, alors $\left(\alpha_{1}, \cdots, \alpha_{n_{1}}\right)$ est aussi une $r$-base canoniquement ordonnée de $K / K_{2}$ (cf. [1], p. 374, proposition 7).

(P9) (Algorithme de la complétion des $r$-bases) Soient $K / k$ une extension purement inséparable finie et $G$ un $r$-générateur de $K / k$. Soit $\left\{\alpha_{1}, \cdots, \alpha_{s}\right\}$ un système de $K$ tel que o $\left(\alpha_{j} / k\left(\alpha_{1}, \cdots, \alpha_{j-1}\right)\right)=o_{j}(K / k)$ $>0$ pour tout $1 \leq j \leq s$. Si $\alpha_{s+1}, \alpha_{s+2}, \cdots$ est une suite d'éléments de $G$ vérifiant $o\left(\alpha_{s+i} / k\left(\alpha_{1}, \cdots, \alpha_{s+i-1}\right)\right)=\max _{a \in G}\left(o\left(a / k\left(\alpha_{1}, \cdots, \alpha_{s+i-1}\right)\right)\right)>$ 0 , alors cette suite s'arrête sur un plus grand entier $n$ tel que o $\left(\alpha_{n}, k\left(\alpha_{1}\right.\right.$, $\left.\left.\cdots, \alpha_{n-1}\right)\right)>0$, et $\left(\alpha_{1}, \cdots, \alpha_{n}\right)$ est une $r$-base canoniquement ordonnée de $K / k$ (cf. [1], p. 374, proposition 8).

\subsection{Extensions modulaires.}

Une extension $K / k$ est dite modulaire, si pour tout $n \in \mathbb{N}, K^{p^{n}}$ et $k$ sont $K^{p^{n}} \cap k$-linéairement disjoints. Cette notion a été introduite pour la première fois par Swedleer dans [5]. Elle caractérise les extensions purement inséparables qui sont produit tensoriel sur $k$ d'extensions simples. Soient $m_{j}$ le $j$-ème exposant de $K / k$ et $\left(\alpha_{1}, \cdots, \alpha_{n}\right)$ une $r$-base canoniquement ordonnée de $K / k$, donc d'après $\left(\mathbf{P}_{\mathbf{7}}\right)$, pour tout $1<j \leq n$, il existe des constantes uniques $C_{\varepsilon} \in k$ telles que $\alpha_{j} p^{m_{j}}=\sum_{\varepsilon \in \Lambda_{j}} C_{\varepsilon}\left(\alpha_{1}, \cdots, \alpha_{j-1}\right)^{m_{j} \varepsilon}$, où $\Lambda_{j}=\left\{\left(i_{1}, \cdots, i_{j-1}\right)\right.$ tel que $\left.0 \leq i_{1}<p^{m_{1}-m_{j}}, \cdots, 0 \leq i_{j-1}<p^{m_{j-1}-m_{j}}\right\}$. Ces relations s'appellent les équations de définition de $K / k$, car elles engendrent l'idéal maximal de toutes les relations entre les $\alpha_{i}, 1 \leq i \leq n$, lequel détermine $K / k$. 
Voici le reste des résultats dont on a besoin :

$\left(\mathbf{P}_{10}\right)$ [Critère du modularité] Sous les notations ci-dessus, les propriétés suivantes sont équivalentes :

1. $K / k$ est modulaire.

2. Pour toute $r$-base canoniquement ordonnée $\left(\alpha_{1}, \cdots, \alpha_{n}\right)$ de $K / k$, les $C_{\varepsilon} \in k \cap K^{m^{m_{j}}}$ pour tout $1<j \leq n$.

3. Il existe une $r$-base canoniquement ordonnée $\left(\alpha_{1}, \cdots, \alpha_{n}\right)$ de $K / k$ telle que les $C_{\varepsilon} \in k \cap K^{p^{m_{j}}}$ pour tout $1<j \leq n$ (cf. [2], p. 142, proposition 1.4).

Ce critère permet de trouver facilement les extensions non modulaires.

Exemple 2.1. Soient $P$ un corps parfait de caractéristique $p>0, k=$ $P(X, Y, Z)$ le corps des fractions rationnelles aux indéterminées $X, Y, Z$, et $K=k\left(\alpha_{1}, \alpha_{2}\right)$ avec $\alpha_{1}=X^{p^{-2}}$ et $\alpha_{2}=X^{p^{-2}} Y^{p^{-1}}+Z^{p^{-1}}$. Il est immédiat que $o_{1}(K / k)=2$ et $o_{2}(K / k)=1\left(\alpha_{2}^{p}=Y \alpha_{1}^{p}+Z\right)$. Si K/k est modulaire, d'après le critère du modularité, $Y^{p^{-1}}$ et $Z^{p^{-1}} \in K$; et donc $K^{\prime}=k\left(X^{p^{-2}}, Y^{p^{-1}}, Z^{p^{-1}}\right) \subset K$. Par suite, di $\left(K^{\prime} / k\right)=3<\operatorname{di}(K / k)=2$, contradiction.

Comme conséquence de ce critère, on a :

$\left(\mathbf{P}_{\mathbf{1 1}}\right)$ Soit $K / k$ une extension purement inséparable finie de degré d'irrationalité $n$. Si $K / k$ est equiexponentielle, alors $K / k$ est modulaire. En particulier, $K / k\left(K^{p^{m_{n}}}\right)$ est equiexponentielle (donc modulaire), où $m_{n}=o_{n}(K / k)$.

$\left(\mathbf{P}_{\mathbf{1 2}}\right)$ Soient $K_{1}$ et $K_{2}$ deux corps intermédiaires d'une extension purement inséparable finie $K / k, k$-linéairement disjoints. Si $K / k$ est modulaire, il existe une $r$-base canoniquement ordonnée $\left(\alpha_{1}, \cdots, \alpha_{n}\right)$ de $K / K_{1}$ telle que $K \simeq K_{1} \otimes_{k} k\left(\alpha_{1}\right) \otimes_{k} \cdots \otimes_{k} k\left(\alpha_{n}\right)$. En particulier, $K / K_{1}$ est modulaire, si $K / k$ l'est.

Le résultat suivant de waterhouse joue un rôle important dans l'étude des extensions modulaires (cf. [6], p. 39, theorem 1.1).

$\left(\mathbf{P}_{\mathbf{1 3}}\right)$ Soit $\left(K_{j}\right)_{j \in I}$ une famille quelconque de sous-corps d'un corps $\Omega$. Si $K$ est un sous-corps de $\Omega$ tel que $K$ et $K_{j}$ sont $K_{j} \cap K$-linéairement disjoints pour tout $j \in I$, alors $K$ et $\bigcap_{j \in I} K_{j}$ sont $K \cap\left(\bigcap_{j \in I} K_{j}\right)$-linéairement disjoints.

Il en résulte aussitôt que la modularité est stable supérieurement et inférieurement par une intersection quelconque. Plus précisément, on a : 
$\left(\mathbf{P}_{14}\right)(i)$ Soit $\left(K_{j} / k\right)_{j \in I}$ une famille de sous-extensions d'une extension $K / k$. Si $K_{j} / k$ est modulaire pour tout $j \in I$, alors $\bigcap_{j \in I} K_{j} / k$ est modulaire.

(ii) Si $K / K_{j}$ est modulaire pour tout $j \in I$, alors $K / \bigcap_{j \in I} K_{j}$ est modulaire. En particulier, $K / k$ admet une plus petite sous-extension $m / k$ (resp. une plus petite extension $M / K$ ) telle que $K / m$ (resp. $M / k$ ) est modulaire.

\section{Résultats principals.}

Soit $K / k$ une extension purement inséparable finie. Une factorisation de longueur $m$ de $K / k$ est la donnée de $m$ corps intermédiaires $K_{1}, K_{2}, \cdots, K_{m}$ de $K / k$ tels que $K \simeq K_{1} \otimes_{k} K_{2} \otimes_{k} \cdots \otimes_{k} K_{m}$. Si de plus, $\left(o_{1}\left(K_{1} / k\right), \cdots, o_{n_{1}}\left(K_{1} /\right.\right.$ $\left.k), o_{1}\left(K_{2} / k\right), \cdots, o_{n_{2}}\left(K_{2} / k\right), \cdots, o_{1}\left(K_{m} / k\right), \cdots, o_{n_{m}}\left(K_{m} / k\right)\right)$ est la liste des exposants de $K / k$, où $n_{j}=d i\left(K_{j} / k\right)$ pour $j=1, \cdots, m$, alors $K_{1} \otimes_{k} K_{2} \otimes_{k}$ $\cdots \otimes_{k} K_{m}$ s'appelle $e$-factorisation de $K / k$. Par récurrence sur $[K: k]$, on voit que $K / k$ admet toujours une factorisation irréductible $\left(K_{i} / k\right.$ irréductible), cependant il n'y a pas unicité de factorisation.

Exemple 3.1. Soient $P$ un corps parfait de caractéristique $p>0, k=$ $k_{0}(X, Y, Z)$ avec $(X, Y, Z)$ algébriquement libre sur $P$, et $K=k\left(X^{p^{-2}}, X^{p^{-2}} Y^{p^{-1}}\right.$ $\left.+Z^{p^{-1}}, Y^{p^{-1}}\right)$. On a

$$
\begin{aligned}
K & \simeq k\left(X^{p^{-2}}, X^{p^{-2}} Y^{p^{-1}}+Z^{p^{-1}}\right) \otimes_{k} k\left(Y^{p^{-1}}\right), \\
& \simeq k\left(X^{p^{-2}}\right) \otimes_{k} k\left(Z^{p^{-1}}\right) \otimes_{k} k\left(Y^{p^{-1}}\right) .
\end{aligned}
$$

$k\left(X^{p^{-2}}, X^{p^{-2}} Y^{p^{-1}}+Z^{p^{-1}}\right) / k$ est irréductible (cf. Exemple 2.1).

Contrairement à l'unicité des facteurs d'une factorisation, le résultat cidessous confirme l'unicité de la forme de la factorisation de longueur maximale d'une certaine classe d'extensions. Plus précisément, on a :

Théorème 3.1. Soit $K / k$ une extension purement inséparable finie de degré d'irrationalité di $(K / k) \leq 4$. Si $K / k$ admet une e-factorisation de longueur maximale $m$, alors toute factorisation de $K / k$ de longueur $m$ est nécessairement une e-factorisation.

Pour la preuve de ce théorème, nous aurons besoin des résultats suivants :

On rencontre fréquemment la proposition ci-dessous dans l'étude de la linéarité disjointe. 
Proposition 3.2. Soient $K_{1}$ et $K_{2}$ deux corps intermédiaires d'une extension $K / k, k$-linéairement disjoints. Soient $L_{1}$ et $L_{2}$ des sous corps respectifs de $K_{1}$ et $K_{2}$. Alors, $L_{2}\left(K_{1}\right)$ et $L_{1}\left(K_{2}\right)$ sont $k\left(L_{1}\right)\left(L_{2}\right)$-linéairement disjoints. En particulier, $L_{2}\left(K_{1}\right) \cap L_{1}\left(K_{2}\right)=k\left(L_{1}\right)\left(L_{2}\right)$.

Preuve. Immédiat, application de la transitivité de la linéarité disjointe.

Ce résultats s'étend à $m$ corps $K_{1}, K_{2}, \cdots, K_{m}, k$-linéairement disjoints.

Soient $L_{i}$ un sous corps de $K_{i}$ pour $i=1, \cdots, m$, et $L=\prod_{i=1}^{m} L_{i}$, alors $L\left(K_{1}\right), L\left(K_{2}\right), \cdots, L\left(K_{m}\right)$ sont $k(L)$-linéairement disjoints.

Proposition 3.3. Soient $K_{1} \otimes_{k} K_{2}$ une factorisation d'une extension purement inséparable finie $K / k$ et $F / k$ la plus petite sous-extension de $K / k$ telle que $K / F$ est modulaire. Si di $\left(K_{1} / k\right) \leq 2$, il existe une sous-extension $K_{1}^{\prime} / k$ de $K / k$ vérifiant :

1. $K \simeq K_{1}^{\prime} \otimes_{k} K_{2}$.

2. $F \cap K_{1}^{\prime}$ est la plus petite sous-extension de $K_{1}^{\prime} / k$ telle que $K_{1}^{\prime} / F \cap K_{1}^{\prime}$ est modulaire.

En particulier, pour tout $i \in \mathbb{N}, o_{i}\left(K_{1}^{\prime} / k\right)=o_{i}\left(K_{1} / k\right)$.

Preuve. On se ramène au cas où $d i\left(K_{1} / k\right)=2$, puisque le cas $\operatorname{di}\left(K_{1} / k\right)=$ 1 est trivial. Posons $m_{1}=o_{1}\left(K_{1} / k\right)$ et $m_{2}=o_{2}\left(K_{1} / k\right)$, et soit $\left(\alpha_{1}, \alpha_{2}\right)$ une $r$-base canoniquement ordonnée de $K_{1} / k$. D'après $\left(\mathbf{P}_{\mathbf{8}}\right)$, on a $\left(\alpha_{1}, \alpha_{2}\right)$ est aussi une $r$-base canoniquement ordonnée de $K / K_{2}$, avec $o_{i}\left(K_{1} / k\right)=$ $o_{i}\left(K / K_{2}\right)$ pour tout $i \in \mathbb{N}$. En vertu de $\left(\mathbf{P}_{11}\right), K / K_{2}\left(\alpha_{1} p^{m_{2}}\right)$ est modulaire, et donc $F \subseteq K_{2}\left(\alpha_{1}{ }^{p^{m_{2}}}\right)$. Posons $s_{1}=o_{1}\left(K_{1}(F) / F\right)$ et $s_{2}=o_{2}\left(K_{1}(F) / F\right)$. D'après $\left(\mathbf{P}_{\mathbf{5}}\right)$ et $\left(\mathbf{P}_{\mathbf{6}}\right), m_{2}=o_{1}\left(K_{1}\left(K_{2}\right) / K_{2}\left(K^{p^{m_{2}}}\right)\right) \leq o_{1}\left(K_{1}\left(K_{2}\right) / K_{2}\right) \leq$ $o_{1}\left(K_{1}(F) / F\right)=s_{1}=o\left(\alpha_{1}, k\left(\alpha_{1} p^{s_{1}}\right)\right)$. De même, on a $m_{2}=o_{2}\left(K / K_{2}\left(K^{p^{m_{2}}}\right)\right)$ $\leq o_{2}\left(K_{1}(F) / F\right)=s_{2} \leq o_{2}\left(K_{1} / k\right)=m_{2}$. On en déduit que $s_{2}=m_{2}$ et $\left[K_{1}\right.$ : $\left.k\left(\alpha_{1} p^{s_{1}}\right)\right]=\left[k\left(\alpha_{1}, \alpha_{2}\right): k\left(\alpha_{1} p^{s_{1}}\right)\right]=\left[k\left(\alpha_{1}, \alpha_{2}\right): k\left(\alpha_{1}\right)\right] \times\left[k\left(\alpha_{1}\right): k\left(\alpha_{1} p^{s_{1}}\right)\right]=$ $p^{m_{2}} p^{s_{1}}=\left[K_{1}(F): F\right]$. Ou encore $K_{1}(F) \simeq K_{1} \otimes_{k\left(\alpha_{1} p^{s_{1}}\right)} F$. Ecrivons l'équation de définition de $K_{1} / k\left(\alpha_{1}{ }^{s_{1}}\right): \alpha_{2}{ }^{p^{m_{2}}}=\sum_{i=0}^{p^{s_{1}-m_{2}}-1} C_{i} \alpha_{1}{ }^{i p^{m_{2}}}$, avec les $C_{i}$ sont uniques dans $k\left(\alpha_{1} p^{s_{1}}\right)$. Or, $\left(\alpha_{1}^{i p^{m_{2}}}\right)_{0 \leq i \leq p^{s_{1}-m_{2}-1}}$ est libre sur $F\left(\operatorname{cf} .\left(\mathbf{P}_{\mathbf{7}}\right)\right)$, donc $\left(\alpha_{1}^{i p^{m_{2}}}\right)_{0 \leq i \leq p^{s_{1}-m_{2}-1}}$ est aussi libre sur $F \cap K^{p^{m_{2}}}$. Prolongeons ce sytème à une base $B$ de $K^{p^{m_{2}}} / F \cap K^{p^{m_{2}}}$. Comme $K^{p^{m_{2}}}$ et $F$ sont $F \cap K^{p^{m_{2}}}$-linéairement disjoints (car $K / F$ est modulaire), alors $B$ est aussi une base de $F\left(K^{p^{m_{2}}}\right) / F$. Puisque les $C_{i} \in k\left(\alpha_{1}{ }^{p_{1}}\right) \subseteq F$, alors par identification les $\left(C_{i}\right)_{0 \leq i \leq p^{s_{1}-m_{2}-1}} \in$ $K^{p^{m_{2}}} \cap F$. Ou encore pour tout $i \in\left\{0, \cdots, p^{s_{1}-m_{2}}-1\right\}, C_{i}^{p^{-m_{2}}} \in K \cap F^{p^{-m_{2}}}$. Par suite, $K=K_{2}\left(\alpha_{1}, \alpha_{2}\right) \subseteq K_{2}\left(\alpha_{1},\left(C_{i}^{p^{-m_{2}}}\right)_{0 \leq i \leq p^{s_{1}-m_{2}-1}}\right) \subseteq K$. D'après 
l'algorithme de la complétion des $r$-bases, il existe $i \in\left\{0, \cdots, p^{s_{1}-m_{2}}-1\right\}$ tel que $K=K_{2}\left(\alpha_{1}, C_{i}^{p^{-m_{2}}}\right)$. Posons $\alpha_{2}^{\prime}=C_{i}{ }^{p^{-m_{2}}}$ et $K_{1}^{\prime}=k\left(\alpha_{1}, \alpha_{2}^{\prime}\right)$. On $\mathrm{a}\left[K_{1}^{\prime}\left(K_{2}\right): K_{2}\right]=\left[K: K_{2}\right]=p^{m_{1}} p^{m_{2}} \leq\left[K_{1}^{\prime}: k\right]=\left[k\left(\alpha_{1}, \alpha_{2}^{\prime}\right): k\right]=$ $\left[k\left(\alpha_{1}, \alpha_{2}^{\prime}\right): k\left(\alpha_{1}\right)\right]\left[k\left(\alpha_{1}\right): k\right] \leq p^{m_{1}} p^{m_{2}},\left(\operatorname{car} \alpha_{2}^{\prime p^{m_{2}}}=C_{i} \in k\left(\alpha_{1} p^{s_{1}}\right) \subseteq k\left(\alpha_{1}\right)\right)$. Il en résulte que $K \simeq K_{1}^{\prime} \otimes_{k} K_{2}$ et $K_{1}^{\prime} \simeq k\left(\alpha_{1}\right) \otimes_{k\left(\alpha_{1} p^{s_{1}}\right)} k\left(\alpha_{1} p^{s_{1}}\right)\left(\alpha_{2}^{\prime}\right)$. On en déduit que la plus petite sous-extension $F_{1}$ de $K_{1}^{\prime} / k$ telle que $K_{1}^{\prime} / F_{1}$ est modulaire est contenue dans $k\left(\alpha_{1}{ }^{s_{1}}\right)$. En particulier, $F_{1} \subseteq F \cap K_{1}^{\prime}$, (car $\left.k\left(\alpha_{1} p^{s_{1}}\right) \subseteq F\right)$. D'autre part, $K \simeq K_{1}^{\prime} \otimes_{k} K_{2} \simeq K_{1}^{\prime} \otimes_{F_{1}} F_{1}\left(K_{2}\right)$, donc $K / F_{1}\left(K_{2}\right)$ est modulaire (car $K_{1}^{\prime} / F_{1}$ est modulaire). D'où $F \subseteq F_{1}\left(K_{2}\right)$, et par suite $F \cap K_{1}^{\prime} \subseteq F_{1}\left(K_{2}\right) \cap K_{1}^{\prime}=F_{1}$ (cf. proposition 3.2). Cela conduit à $F_{1}=F \cap K_{1}^{\prime}$.

D'après l'associativité et la commutativité du produit tensoriel, le résultat ci-dessus se généralise à une factorisation de $m$ facteurs comme suit :

Proposition 3.4. Soient $K_{1} \otimes_{k} \cdots \otimes_{k} K_{m}$ une factorisation d'une extension purement inséparable finie $K / k$, et $F / k$ la plus petite sous-extension de $K / k$ telle que $K / F$ est modulaire. Si pour tout $j \in\{1, \cdots, m\}$, di $\left(K_{j} / k\right) \leq 2$, alors il existe une famille $\left(K_{i}^{\prime}\right)_{1 \leq i \leq m}$ de sous-extensions de $K / k$ vérifiant :

1. $K \simeq K_{1}^{\prime} \otimes_{k} \cdots \otimes_{k} K_{m}^{\prime}$.

2. $F \cap K_{i}^{\prime}$ est la plus petite sous-extension de $K_{i}^{\prime} / k$ telle que $K_{i}^{\prime} / F \cap K_{i}^{\prime}$ est modulaire pour $i=1,2, \cdots, m$.

3. Pour tout $j \in\{1, \cdots, m\}$, pour tout $i \in \mathbb{N}, o_{i}\left(K_{j}^{\prime} / k\right)=o_{i}\left(K_{j} / k\right)$. En particulier, $F \simeq F \cap K_{1}^{\prime} \otimes_{k} \cdots \otimes_{k} F \cap K_{m}^{\prime}$, et donc $F / k$ est modulaire.

Preuve. Immédiat, application de la proposition 3.2 et la proposition 3.3.

Lemme 3.5. Soit $\left(\alpha_{1}, \cdots, \alpha_{n}\right)$ une r-base canoniquement ordonnée d'une extension purement inséparable finie $K / k$. S'il existe $i \in\{1, \cdots, n\}$ tel que $K \simeq k\left(\alpha_{1}, \cdots, \alpha_{i}\right) \otimes_{k} k\left(\alpha_{i+1}, \cdots, \alpha_{n}\right)$, alors pour toute partie $\left\{\alpha_{1}^{\prime}, \cdots, \alpha_{i}^{\prime}\right\}$ de $K$ telle que $\left\{\alpha_{1}^{\prime}, \cdots, \alpha_{i}^{\prime}, \alpha_{i+1}, \cdots, \alpha_{n}\right\}$ est une $r$-base de $K / k$, on a $K \simeq$ $k\left(\alpha_{1}^{\prime}, \cdots, \alpha_{i}^{\prime}\right) \otimes_{k} k\left(\alpha_{i+1}, \cdots, \alpha_{n}\right)$. En outre, $o_{j}(K / k)=o_{j}\left(k\left(\alpha_{1}^{\prime}, \cdots, \alpha_{i}^{\prime}\right) / k\right)$ pour $j=1,2 \cdots, i$

Preuve. cf. [1].

Comme conséquence immédiate, on a :

Proposition 3.6. Soit $\left(\alpha_{1}, \cdots, \alpha_{n}\right)$ (resp. $G$ ) une $r$-base canoniquement ordonnée (resp. $r$-base) d'une extension purement inséparable finie $K / k$. On suppose que $K \simeq k\left(\alpha_{1}, \cdots, \alpha_{i}\right) \otimes_{k} k\left(\alpha_{i+1}, \cdots, \alpha_{n}\right)$. Alors, il existe $\left\{\alpha_{1}^{\prime}, \cdots\right.$, $\left.\alpha_{i}^{\prime}\right\} \subseteq G$ telle que $\left(\alpha_{1}^{\prime}, \cdots, \alpha_{i}^{\prime}, \alpha_{i+1}, \cdots, \alpha_{n}\right)$ est une $r$-base canoniquement ordonnée de $K / k$, et on a $K \simeq k\left(\alpha_{1}^{\prime}, \cdots, \alpha_{i}^{\prime}\right) \otimes_{k} k\left(\alpha_{i+1}, \cdots, \alpha_{n}\right)$. 
Preuve. Application du lemme 3.5 et de l'algorithme de la complétion des $r$-bases.

Preuve du théorème 3.1. On présente la démonstration uniquement dans le cas où $d i(K / k)=4$ et $K / k$ admet une $e$-factorisation de longueur maximale $m=2$, puisque les autres situations ou bien elles sont triviales, ou bien elles ont des preuves analogues à ce cas.

Etant données une extension purement inséparable finie $K / k$ de degré d'irrationalité 4 et $\left(\alpha_{1}, \cdots, \alpha_{4}\right)$ (resp. $\left.\left\{u_{1}, \cdots, u_{4}\right\}\right)$ une $r$-base canoniquement ordonnée (resp. une $r$-base) de $K / k$. Soit $F / k$ la plus petite sousextension de $K / k$ telle que $K / F$ est modulaire.

1-ière étape $: \mathbf{K} \simeq \mathbf{k}\left(\alpha_{1}\right) \otimes_{\mathbf{k}} \mathbf{k}\left(\alpha_{2}, \alpha_{3}, \alpha_{\mathbf{4}}\right)$.

1-ier cas : $K \simeq k\left(u_{1}, u_{2}\right) \otimes_{k} k\left(u_{3}, u_{4}\right)$. Posons $L_{1}=k\left(u_{1}, u_{2}\right)$ et $L_{2}=$ $k\left(u_{3}, u_{4}\right)$. D'après la proposition 3.4 , on se ramène au cas où $L_{1} / F \cap L_{1}$ et $L_{2} / F \cap L_{2}$ sont modulaires. Comme $K / k\left(\alpha_{2}, \alpha_{3}, \alpha_{4}\right)$ est simple (donc modulaire), il en résulte que $F \subseteq k\left(\alpha_{2}, \alpha_{3}, \alpha_{4}\right)$.

Par suite, $o_{1}(K / k)=o\left(\alpha_{1} / k\left(\alpha_{2}, \alpha_{3}, \alpha_{4}\right)\right) \leq o\left(\alpha_{1} / F\right) \leq o_{1}(K / k)\left(\mathrm{cf} .\left(\mathbf{P}_{6}\right)\right)$, d'où $o_{1}(K / F)=o_{1}(K / k)$. On en déduit que $o_{1}\left(L_{1} / L_{1} \cap F\right)=o_{1}(K / k)$ ou $o_{1}\left(L_{2} / L_{2} \cap F\right)=o_{1}(K / k)$, puisque $o_{1}(K / k)=o_{1}(K / F)=o_{1}\left(L_{1}\left(L_{2}\right) / F\right)=$ $\sup \left(o_{1}\left(F\left(L_{1}\right) / F\right), o_{1}\left(F\left(L_{2}\right) / F\right)\right) \leq \sup \left(o_{1}\left(L_{1} / L_{1} \cap F\right), o_{1}\left(L_{2} / L_{2} \cap F\right)\right) \leq$ $o_{1}(K / k)$. Ou encore $L_{1} \cap F=k$ ou $L_{2} \cap F=k$. D'où $K / k$ admet une factorisation de longueur 3 , (car $L_{1} / k$ ou $L_{2} / k$ serait modulaire), ce qui contredit l'hypothèse, donc ce cas ne peut exister.

2-ième cas : $K \simeq k\left(u_{1}\right) \otimes k\left(u_{2}, u_{3}, u_{4}\right)$. Posons $L_{1}=k\left(u_{1}\right)$ et $L_{2}=k\left(u_{2}, u_{3}\right.$, $\left.u_{4}\right)$. Puisque $K / L_{2}$ est modulaire $\left(K / L_{2}\right.$ est simple), alors $F \subseteq L_{2}$. D'après la proposition 3.2, on obtient $K \simeq L_{1} \otimes_{k} L_{2} \simeq F\left(L_{1}\right) \otimes_{F} L_{2}$; et d'après $\left(\mathbf{P}_{\mathbf{1 2}}\right)$, il existe une $r$-base canoniquement ordonnée $\left(\alpha_{1}^{\prime}, \alpha_{2}^{\prime}, \alpha_{3}^{\prime}\right)$ de $K / F\left(L_{1}\right)$ telle que $K \simeq F\left(L_{1}\right) \otimes_{F} F\left(\alpha_{1}^{\prime}\right) \otimes_{F} F\left(\alpha_{2}^{\prime}\right) \otimes_{F} F\left(\alpha_{3}^{\prime}\right)$. D'autre part, on a $K / k\left(\alpha_{2}, \alpha_{3}, \alpha_{4}\right)$ est simple (donc modulaire), il en résulte que $F \subseteq k\left(\alpha_{2}, \alpha_{3}\right.$, $\left.\alpha_{4}\right)$. D'où $o_{1}(K / k)=o_{1}\left(K / k\left(\alpha_{2}, \alpha_{3}, \alpha_{4}\right)\right) \leq o_{1}(K / F) \leq o_{1}(K / k)$, donc $o_{1}(K / F)=o_{1}(K / k)$. Si $o_{1}\left(L_{1}(F) / F\right)<o_{1}(K / k)$, alors $o\left(\alpha_{1}^{\prime} / F\right)=o_{1}(K / k)$ $=o\left(\alpha_{1}^{\prime} / k\right)$. En vertu du $\left(\mathbf{P}_{\mathbf{8}}\right), F\left(\alpha_{1}^{\prime}\right)=k\left(\alpha_{1}^{\prime}\right) \otimes_{k} F$. On en déduit que $K \simeq F\left(L_{1}\right) \otimes_{F} F\left(\alpha_{1}^{\prime}\right) \otimes_{F} F\left(\alpha_{2}^{\prime}\right) \otimes_{F} F\left(\alpha_{3}^{\prime}\right) \simeq\left(L_{1} \otimes_{k} F\right) \otimes_{F}\left[\left(k\left(\alpha_{1}^{\prime}\right) \otimes_{k} F\right) \otimes_{F}\right.$ $\left.F\left(\alpha_{2}^{\prime}, \alpha_{3}^{\prime}\right)\right] \simeq L_{1} \otimes_{k} k\left(\alpha_{1}^{\prime}\right) \otimes_{k} F\left(\alpha_{2}^{\prime}, \alpha_{3}^{\prime}\right)$. D'où $K / k$ admet une factorisation de longueur 3, contradiction. Par suite, $o_{1}\left(L_{1}(F) / F\right)=o_{1}\left(L_{1} / k\right)=o_{1}(K / k)$, c'est-à-dire $L_{1} \otimes_{k} L_{2}$ est aussi une $e$-factorisation.

2-ième étape $: \mathbf{K} \simeq \mathbf{k}\left(\alpha_{1}, \alpha_{2}\right) \otimes_{\mathbf{k}} \mathbf{k}\left(\alpha_{3}, \alpha_{4}\right)$.

1-ier cas : $K \simeq k\left(u_{1}, u_{2}\right) \otimes_{k} k\left(u_{3}, u_{4}\right)$. Posons $L_{1}=k\left(u_{1}, u_{2}\right)$ et $L_{2}=$ $k\left(u_{3}, u_{4}\right)$. D'après la proposition 3.6, il existe $u_{i}, u_{j} \in\left\{u_{1}, u_{2}, u_{3}, u_{4}\right\}$ telle que $K \simeq k\left(u_{i}, u_{j}\right) \otimes_{k} k\left(\alpha_{3}, \alpha_{4}\right)$. Nécessairement $\{i, j\}=\{1,2\}$ ou $\{i, j\}=\{3,4\}$, sinon $K / k$ admet une factorisation de longueur 3 . Il en résulte que $L_{1} \otimes_{k} L_{2}$ est aussi une $e$-factorisation. 
2-ième cas : $K \simeq k\left(u_{1}\right) \otimes_{k} k\left(u_{2}, u_{3}, u_{4}\right)$. D'après la proposition 3.6, il existe $\left\{u_{i}, u_{j}\right\} \subseteq\left\{u_{1}, u_{2}, u_{3}, u_{4}\right\}$ telle que $\left.K \simeq k\left(u_{i}, u_{j}\right) \otimes_{k} k\left(\alpha_{2}, \alpha_{3}\right)\right)$. Nécessairement $\left\{u_{i}, u_{j}\right\} \subseteq\left\{u_{2}, u_{3}, u_{4}\right\}$, sinon $K / k$ admet une factorisation de longueur 3 , (puisque $k\left(u_{1}, u_{j}\right)=k\left(u_{1}\right) \otimes_{k} k\left(u_{j}\right)$ pour tout $2 \leq j \leq 4$ ). Or, $K \simeq$ $k\left(u_{1}\right) \otimes_{k} k\left(u_{2}, u_{3}, u_{4}\right) \simeq k\left(u_{1}, u_{i}, u_{j}\right) \otimes_{k\left(u_{i}, u_{j}\right)} k\left(u_{2}, u_{3}, u_{4}\right)$, donc $K / k\left(u_{i}, u_{j}\right)$ est modulaire. D'après la propriété $\left(\mathbf{P}_{\mathbf{1 2}}\right)$ appliquée à $K \simeq k\left(u_{i}, u_{j}\right) \otimes_{k}$ $k\left(\alpha_{3}, \alpha_{4}\right) / k\left(u_{i}, u_{j}\right)$, on se ramène au cas où $K \simeq k\left(u_{i}, u_{j}\right) \otimes_{k} k\left(\alpha_{1}^{\prime}\right) \otimes_{k} k\left(\alpha_{2}^{\prime}\right)$, avec $\left\{\alpha_{1}^{\prime}, \alpha_{2}^{\prime}\right\}$ est une $r$-base de $K / k\left(u_{i}, u_{j}\right)$ (contradiction), donc ce cas ne peut exister.

3-ième étape : $\mathbf{k}\left(\alpha_{1}, \alpha_{2}, \alpha_{3}\right) \otimes_{\mathbf{k}} \mathbf{k}\left(\alpha_{4}\right)$.

1-ier cas : $K \simeq k\left(u_{1}\right) \otimes_{k} k\left(u_{2}, u_{3}, u_{4}\right)$. D'après la proposition 3.6, il existe $u_{i}, u_{j}, u_{h} \in\left\{u_{1}, u_{2}, u_{3}, u_{4}\right\}$ telle que $K \simeq k\left(u_{i}, u_{j}, u_{h}\right) \otimes_{k} k\left(\alpha_{4}\right)$. Nécessairement $\left\{u_{i}, u_{j}, u_{h}\right\}=\left\{u_{2}, u_{3}, u_{4}\right\}$, sinon $K / k$ admet une factorisation de longueur 3. On en déduit que $k\left(u_{1}, u_{2}, u_{3}\right) \otimes_{k} k\left(u_{4}\right)$ est aussi une $e$-factorisation. 2-ième cas : $K \simeq k\left(u_{1}, u_{2}\right) \otimes_{k} k\left(u_{3}, u_{4}\right)$. Il existe $u_{i}, u_{j}, u_{h} \in\left\{u_{1}, u_{2}, u_{3}, u_{4}\right\}$ telle que $K \simeq k\left(u_{i}, u_{j}, u_{h}\right) \otimes_{k} k\left(\alpha_{4}\right)$. Il en résulte que $K / k$ admet une factorisation de longueur 3 (car on peut supposer que $k\left(u_{i}, u_{j}, u_{h}\right)=k\left(u_{1}, u_{2}, u_{3}\right) \simeq$ $\left.k\left(u_{1}, u_{2}\right) \otimes_{k} k\left(u_{3}\right)\right)$.

Remarque 3.1. D'après la démonstration du théorème ci-dessus, les facteurs d'une e-factorisation de longueur maximale d'une extension purement inséparable finie $K / k$ de degré d'irrationalité di $(K / k) \leq 4$ conservent leurs tailles et leurs formes, donc on a unicité de la forme d'un certain type de factorisation.

Comme conséquence immédiate on a :

Corollaire 3.7. Soit $K / k$ une extension purement inséparable finie de degré d'irrationalité vérifiant $2<\operatorname{di}(K / k) \leq 4$. Si $K / k$ admet deux types différents de factorisations de longueur $m$ dont l'une est une e-factorisation, alors $K / k$ admet une factorisation de longueur $m+1$.

Preuve. Immédiat.

En particulier, on a :

Corollaire 3.8. Soit $K / k$ une extension purement inséparable finie de degré d'irrationalité vérifiant $2<d i(K / k) \leq 4$. Si $K / k$ admet deux types différents de factorisations de longueur di $(K / k)-1$ dont l'une est une e-factorisation, alors $K / k$ est modulaire.

Preuve. Immédiat. 


\subsection{Contre-exemple.}

Contrairement aux extensions purement inséparables finies de degré d'irrationalité majoré par 4, voici un contre-exemple d'extension qui ne conserve ni la taille ni la forme des facteurs de ces factorisations de longueur maximale.

Exemple 3.2. Soient $k_{0}$ un corps parfait de caractéristique $p>0$ et $X, Y, Z$, $\lambda_{0}, \lambda_{1}, \lambda_{2}$ algébriquement indépendants sur $k_{0}$. Soit $k=k_{0}\left(X, Y, Z, \lambda_{0}, \lambda_{1}, \lambda_{2}\right)$ le corps du base. Posons $K=k\left(X^{p^{-2}}, Y^{p^{-3}}, \lambda_{0}{ }^{p^{-1}}+\lambda_{1}{ }^{p^{-1}} X^{p^{-2}}+\lambda_{2}{ }^{p^{-1}} Y^{p^{-2}}\right.$, $\left.Z^{p^{-3}}, \lambda_{0}{ }^{p^{-1}}+\lambda_{1}^{p^{-1}} Z^{p^{-3}}\right), F=k\left(\frac{X^{p^{-1}}}{Y^{p^{-1}}}-\frac{1}{Y^{p^{-1}}} Z^{p^{-2}}, Z^{p^{-2}}\right), L_{1}=k\left(Y^{p^{-3}}\right.$, $\left.X^{p^{-2}}, \lambda_{0}{ }^{p^{-1}}+\lambda_{1}{ }^{p^{-1}} X^{p^{-2}}+\lambda_{2}{ }^{p^{-1}} Y^{p^{-2}}\right), L_{2}=k\left(Z^{p^{-3}}, \lambda_{0}^{p^{-1}}+\lambda_{1}{ }^{p^{-1}} Z^{p^{-3}}\right), L_{1}^{\prime}=$ $k\left(Y^{p^{-3}}\right)$, et $L_{2}^{\prime}=k\left(Z^{p^{-3}}, \frac{X^{p^{-2}}-Z^{p^{-3}}}{Y^{p^{-2}}}, \lambda_{1}^{p^{-1}} \frac{X^{p^{-2}}-Z^{p^{-3}}}{Y^{p^{-2}}}+\lambda_{2}^{p^{-1}}, \lambda_{0}^{p^{-1}}+\right.$ $\left.\lambda_{1}^{p^{-1}} Z^{p^{-3}}\right)$.

Théorème 3.9. Sous les notations ci-dessus, on a $L_{1} \otimes_{k} L_{2}$ et $L_{1}^{\prime} \otimes_{k} L_{2}^{\prime}$ sont deux factorisations de types différents de $K / k$ de longueur maximale 2 , avec $L_{1}^{\prime} \otimes_{k} L_{2}^{\prime}$ est une e-factorisation.

Pour la preuve de ce théorème, nous aurons besoin des résultats suivants.

Lemme 3.10. $F=k\left(\frac{X^{p^{-1}}}{Y^{p^{-1}}}-\frac{1}{Y^{p^{-1}}} Z^{p^{-2}}, Z^{p^{-2}}\right)$ est la plus petite sous-extension de $K / k$ telle que $K / F$ est modulaire.

Preuve. Pour alléger l'écriture on pose $\theta_{1}=Y^{p^{-3}}, \theta_{2}=Z^{p^{-3}}, \theta_{3}=$ $\frac{X^{p^{-2}}-Z^{p^{-3}}}{Y^{p^{-2}}}, \theta_{4}=\lambda_{1}{ }^{p^{-1}} \frac{X^{p^{-2}}-Z^{p^{-3}}}{Y^{p^{-2}}}+\lambda_{2}{ }^{p^{-1}}$ et $\theta_{5}=\lambda_{0}{ }^{p^{-1}}+\lambda_{1}{ }^{p^{-1}} Z^{p^{-3}} \cdot \mathrm{Si}$ $\theta_{3}{ }^{p} \notin F$, donc $\left(1, \theta_{3}{ }^{p}\right)$ est libre sur $F \cap K^{p}$. Prolongeons ce système à une base $B$ de $K^{p} / F \cap K^{p}$. Comme $\theta_{4}{ }^{p}=\lambda_{1} \theta_{3}{ }^{p}+\lambda_{2}$, et comme $K^{p}$ et $F$ sont $F \cap K^{p}$ linéairement disjoints, (car $K / F$ est modulaire), alors $B$ est aussi une base de $F\left(K^{p}\right) / F$. Par identification, $\lambda_{1}{ }^{p^{-1}}, \lambda_{2}{ }^{p^{-1}} \in K$; et par suite $\lambda_{0}{ }^{p^{-1}} \in K$ $\left(\right.$ car $\left.\lambda_{0}{ }^{p^{-1}}+\lambda_{1}{ }^{p^{-1}} Z^{p^{-3}} \in K\right)$. D'où on aura :

$$
d_{i}\left(k\left(X^{p^{-1}}, Z^{p^{-1}}, Y^{p^{-1}}, \lambda_{0}{ }^{p^{-1}}, \lambda_{1}{ }^{p^{-1}}, \lambda_{2}{ }^{p^{-1}}\right) / k\right)=6 \leq d_{i}(K / k)=5,
$$

ce qui est absurde. De la même façon on montre que $Z^{p^{-2}} \in F$. D'autre part, on a $K / k\left(\frac{X^{p^{-1}}}{Y^{p^{-1}}}-\frac{1}{Y^{p^{-1}}} Z^{p^{-2}}, Z^{p^{-2}}\right)$ est modulaire, donc $F=k\left(\frac{X^{p^{-1}}}{Y^{p^{-1}}}-\right.$ $\left.\frac{1}{Y^{p^{-1}}} Z^{p^{-2}}, Z^{p^{-2}}\right)$.

Lemme 3.11. $k_{1}=k\left(Z^{p^{-1}}\right)$ est la plus petite sous-extension de $F / k$ telle que $F / k_{1}$ est modulaire. En particulier, $F / k$ est non modulaire. 
Preuve. Démonstration analogue à celle du lemme précédent. Il suffit d'appliquer le critère du modularité.

Lemme 3.12. Soit $\left(a_{1}^{\prime}, \cdots, a_{n}^{\prime}\right)$ une $r$-base canoniquement ordonnée d'une extension purement inséparable finie $K / k$ vérifiant $K \simeq k\left(a_{1}^{\prime}, \cdots, a_{t-1}^{\prime}\right) \otimes_{k}$ $k\left(a_{t}^{\prime}, \cdots, a_{n}^{\prime}\right)$. Si $\left(a_{1}, \cdots, a_{n}\right)$ est une $r$-base canoniquement ordonnée de $K / k$, et s'il existe $s \in\{1, \cdots, t-1\}$ tel que $o_{s}(K / k)>o_{t}(K / k)$, alors $\left\{a_{1}, \cdots, a_{s}\right.$, $\left.a_{t}^{\prime}, \cdots, a_{n}^{\prime}\right\}$ est $r$-libre sur $k\left(K^{p}\right)$.

Preuve. cf. [1].

Preuve du théorème 3.9. Supposons que $K / k$ admet une factorisation de longueur meilleure que 2. Compte tenu de l'associativité et la commutativité du produit tensoriel (déplacement et regroupement des facteurs), on se ramène au cas où $K \simeq K_{1}^{\prime} \otimes_{k} K_{2}^{\prime} \otimes_{k} K_{3}^{\prime}$, avec $\operatorname{di}\left(K_{1}^{\prime} / k\right) \geq \operatorname{di}\left(K_{2}^{\prime} / k\right) \geq$ $\operatorname{di}\left(K_{3}^{\prime} / k\right)$. Si $\operatorname{di}\left(K_{1}^{\prime} / k\right)=2$, alors $\operatorname{di}\left(K_{2}^{\prime} / k\right)=2$ et $\operatorname{di}\left(K_{3}^{\prime} / k\right)=1$. D'après la proposition 3.4, on aura $F / k$ est modulaire, ce qui absurde. Cela se traduit par $\operatorname{di}\left(K_{1}^{\prime} / k\right)=3$ et $d i\left(K_{2}^{\prime} / k\right)=d_{i}\left(K_{3}^{\prime} / k\right)=1$. Comme $K \simeq K_{1}^{\prime}\left(K_{2}^{\prime}\right) \otimes_{K_{1}^{\prime}}$ $K_{1}^{\prime}\left(K_{3}^{\prime}\right)$ et $\operatorname{di}\left(K_{2}^{\prime}\left(K_{1}^{\prime}\right) / K_{1}^{\prime}\right)=\operatorname{di}\left(K_{3}^{\prime}\left(K_{1}^{\prime}\right) / K_{1}^{\prime}\right)=1$, il en résulte que $K / K_{1}^{\prime}$ est modulaire. D'où $F \subseteq K_{1}^{\prime}$, et en particulier $K \simeq K_{1}^{\prime} \otimes_{F} F\left(K_{2}^{\prime}\right) \otimes_{F} F\left(K_{3}^{\prime}\right)$. Or, $o_{1}(K / F)=3$ et $o_{2}(K / F)=o_{3}(K / F)=o_{4}(K / F)=o_{5}(K / F)=1$, donc $o_{1}\left(K_{2}^{\prime}(F) / F\right)=o_{1}\left(K_{2}^{\prime} / k\right)=1$ ou $o_{1}\left(K_{3}^{\prime}(F) / F\right)=o_{1}\left(K_{3}^{\prime} / k\right)=1$. Ainsi, on se place dans la cas : $K \simeq k\left(\alpha_{1}, \cdots, \alpha_{4}\right) \otimes_{k} k\left(\alpha_{5}\right)$ où $\left(\alpha_{1}, \cdots, \alpha_{5}\right)$ est une $r$-base canoniquement ordonnée de $K / k$. En vertu du lemme 3.12, $\left\{Z^{p^{-3}}, Y^{p^{-3}}, X^{p^{-2}}, \alpha_{5}\right\}$ est $r$-libre sur $k\left(K^{p}\right)$. D'après l'algorithme de la complétion des $r$-bases appliqué à $K / k\left(K^{p}\right)$, deux cas peuvent se produire. Notons bien que $G=\left\{Z^{p^{-3}}, Y^{p^{-3}}, X^{p^{-2}}, \lambda_{0}{ }^{p^{-1}}+\lambda_{1}{ }^{p^{-1}} Z^{p^{-2}}, \lambda_{0}{ }^{p^{-1}}+\lambda_{1}{ }^{p^{-1}} X^{p^{-2}}+\right.$ $\left.\lambda_{2}{ }^{p^{-1}} Y^{p^{-2}}\right\}$ est une $r$-base de $K / k$.

1-ier cas : $\left\{Z^{p^{-3}}, Y^{p^{-3}}, X^{p^{-2}}, \alpha_{5}^{\prime}, \lambda_{0}{ }^{p^{-1}}+\lambda_{1}{ }^{p^{-1}} Z^{p^{-2}}\right\}$ est une $r$-base de $K / k($ $\left.K^{p}\right)$. D'après $\left(\mathbf{P}_{\mathbf{1}}\right),\left\{Z^{p^{-3}}, Y^{p^{-3}}, X^{p^{-2}}, \alpha_{5}^{\prime}, \lambda_{0}{ }^{p^{-1}}+\lambda_{1}{ }^{p^{-1}} Z^{p^{-2}}\right\}$ est aussi une $r$-base de $K / k$, et on a

$$
\begin{gathered}
K \simeq k\left(Y^{p^{-3}}\right) \otimes_{k} k\left(X^{p^{-2}}\right) \otimes_{k} k\left(Z^{p^{-3}}, \lambda_{0}^{p^{-1}}+\lambda_{1}^{p^{-1}} Z^{p^{-2}}\right) \otimes_{k} k\left(\alpha_{5}^{\prime}\right) \simeq \\
\simeq k\left(Z^{p^{-3}}\right)\left(Y^{p^{-3}}\right) \otimes_{k\left(Z^{p^{-3}}\right)} k\left(Z^{p^{-3}}\right)\left(X^{p^{-2}}\right) \otimes_{k\left(Z^{p^{-3}}\right)} k\left(Z^{p^{-3}}\right)\left(\lambda_{0}^{p^{-1}}+\lambda_{1}^{p^{-1}} Z^{p^{-2}}\right) \\
\otimes_{k\left(Z^{p^{-3}}\right)} k\left(Z^{p^{-3}}\right)\left(\alpha_{5}^{\prime}\right) .
\end{gathered}
$$

On en déduit que $K / k\left(Z^{p^{-3}}\right)$ est modulaire, d'où $F \subseteq k\left(Z^{p^{-3}}\right)$. Par suite, $2=\operatorname{di}(F / k) \leq \operatorname{di}\left(k\left(Z^{p^{-3}}\right) / k\right)=1$, ce qui absurde.

2-ième cas : $\left\{Z^{p^{-3}}, Y^{p^{-3}}, X^{p^{-2}}, \lambda_{0}{ }^{p^{-1}}+\lambda_{1}{ }^{p^{-1}} X^{p^{-2}}+\lambda_{2}{ }^{-1} Y^{p^{-2}}, \alpha_{5}^{\prime}\right\}$ est une $r$-base de $K / k$. D'où $K \simeq k\left(Z^{p^{-3}}\right) \otimes_{k} k\left(Y^{p^{-3}}, X^{p^{-2}}, \lambda_{0}{ }^{p^{-1}}+\lambda_{1}{ }^{p^{-1}} X^{p^{-2}}+\right.$ $\left.\lambda_{2}{ }^{p^{-1}} Y^{p^{-2}}\right) \otimes_{k} k\left(\alpha_{5}^{\prime}\right)$. On vérifie aussitôt que $K / k\left(X^{p^{-1}}, Y^{p^{-1}}\right)$ est modulaire, 
et par conséquent $F \subseteq k\left(X^{p^{-1}}, Y^{p^{-1}}\right)$. D'après $\left(\mathbf{P}_{\mathbf{6}}\right)$, on obtient $o_{1}(F / k)=$ $2 \leq o_{1}\left(k\left(X^{p^{-1}}, Y^{p^{-1}}\right) / k\right)=1$, contradiction.

\section{Références}

[1] M. Chellali et E. Fliouet, Sur les extensions purement inséparable, Arch. Math., 41, (2003), 369-382

[2] M. Chellali et E. Fliouet, Extensions purement inséparables d'exposant non borné, Archivum Mathematicum, 40, (2004), 129-159

[3] J.N. Mordeson and B.Vinograde, Structure of arbitrary purely inseparable extension fields, Springer-Verlag, Berlin, LNM 173, 1973

[4] G. Pickert, Inseparable Körperweiterungen, Math. Z., 52, (1949), 81-135

[5] M.E. Sweedler, Structure of inseparable extensions, Ann. Math., 87, (1968), 401410

[6] W.C. Waterhouse, The structure of inseparable field extensions, Trans. Am. Math. Soc., 211, (1975), 39-56

El Hassane Fliouet

Département de mathématiques

Faculté des sciences

Université Mohammed 1, Oujda

Maroc

E-mail: fliouet@yahoo.fr

Received: 19.04.2014

Accepted: 20.09.2014 